\title{
Reduced conditioned fear response in mice that lack Dlx 1 and show subtype-specific loss of interneurons
}

\author{
Rong Mao • Damon T. Page • Irina Merzlyak • \\ Carol Kim • Laurence H. Tecott • Patricia H. Janak • \\ John L. R. Rubenstein • Mriganka Sur
}

Received: 14 February 2009 / Accepted: 23 June 2009 /Published online: 11 July 2009

(C) The Author(s) 2009. This article is published with open access at Springerlink.com

\begin{abstract}
The inhibitory GABAergic system has been implicated in multiple neuropsychiatric diseases such as schizophrenia and autism. The $D l x$ homeobox transcription factor family is essential for development and function of GABAergic interneurons. Mice lacking the Dlx1 gene have postnatal subtype-specific loss of interneurons and reduced IPSCs in their cortex and hippocampus. To ascertain consequences of these changes in the GABAergic system, we performed a battery of behavioral assays on the Dlx 1 mutant mice, including zero maze, open field, locomotor activity, food intake, rotarod, tail suspension, fear conditioning assays (context and trace), prepulse inhibition, and working memory related tasks (spontaneous alteration task and spatial working memory task). Dlx 1 mutant mice displayed elevated activity levels in open field, locomotor activity, and tail suspension tests. These mice also showed
\end{abstract}

Electronic supplementary material The online version of this article (doi:10.1007/s11689-009-9025-8) contains supplementary material, which is available to authorized users.

R. Mao $(\bowtie) \cdot$ D. T. Page $\cdot$ M. Sur $(\bowtie)$

Picower Institute for Learning and Memory,

Massachusetts Institute of Technology,

Cambridge, MA 02139, USA

e-mail: rmao@mit.edu

e-mail: msur@mit.edu

R. Mao $\cdot$ D. T. Page $\cdot$ M. Sur

Department of Brain and Cognitive Sciences,

Massachusetts Institute of Technology,

Cambridge, MA 02139, USA

I. Merzlyak • P. H. Janak

Ernest Gallo Clinic and Research Center, University of California,

San Francisco,

Emeryville, CA 94608, USA deficits in contextual and trace fear conditioning, and possibly in prepulse inhibition. Their learning deficits were not global, as the mutant mice did not differ from the wildtype controls in tests of working memory. Our findings demonstrate a critical role for the Dlxl gene, and likely the subclasses of interneurons that are affected by the lack of this gene, in behavioral inhibition and associative fear learning. These observations support the involvement of particular components of the GABAergic system in specific behavioral phenotypes related to complex neuropsychiatric diseases.

Keywords Behavior · Fear conditioning - Associative learning · Prepulse inhibition · Hyperactivity · Interneuron · Inhibitory · GABAergic · Calretinin ·

Neuropsychiatric disease

C. Kim • J. L. R. Rubenstein

Nina Ireland Laboratory of Developmental Neurobiology, Center for Neurobiology and Psychiatry, Department of Psychiatry,

University of California, San Francisco,

San Francisco, CA 94158, USA

\section{H. Tecott}

Department of Psychiatry, University of California, San Francisco, San Francisco, CA 94158-2822, USA

P. H. Janak

Department of Neurology, University of California, San Francisco, CA 94143, USA

P. H. Janak

Wheeler Center for the Neurobiology of Addiction, University of California,

San Francisco, CA 94143, USA 


$\begin{array}{ll}\text { Abbreviations } \\ \text { ITI } & \text { inter-trial interval } \\ \text { CS } & \text { conditioned stimulus } \\ \text { US } & \text { unconditioned stimulus } \\ \text { SPL } & \text { sound pressure level } \\ \text { PFC } & \text { prefrontal cortex } \\ \text { mPFC } & \text { medial prefrontal cortices } \\ \text { DH } & \text { dorsal hippocampus } \\ \text { VH } & \text { ventral hippocampus }\end{array}$

\section{Introduction}

GABAergic inhibitory interneurons are vital for cortical development and function $[1,2]$. They can be distinguished from pyramidal cells by their smooth or sparsely spiny dendrites and locally projecting axons. Interneurons are vastly heterogeneous in their morphological, physiological, molecular and synaptic characteristics, despite the fact that this cell population comprises only $20-30 \%$ of cortical neurons. Interneuron deficits are implicated in neuropsychiatric disorders including schizophrenia [3, 4], autism [5, 6], anxiety [7, 8], and mood disorders [9-11]. However, our understanding of the development and function of these cells is limited.

The family of $D l x$ homeobox transcription factors regulates the development of inhibitory interneurons; members of this family of genes, namely Dlx1, Dlx2, Dlx5, and Dlx6, control differentiation of GABAergic neurons in the basal ganglia and cerebral cortex [12-17]. $D l x 1$ is essential for maintaining the functional longevity of interneurons in the adult brain: homozygous $D l x l$ knockout mice $\left(D l x 1^{-/-}\right)$show partial loss of specific subsets of postnatal neocortical and hippocampal interneurons as a result of apoptosis, along with functional onset of epilepsy [18].

Given the importance of the $D l x l$ gene in interneuron development and survival, $D l x l$ knockout mice provide an opportunity to probe whether loss of these neurons results in behavioral phenotypes that present in neuropsychiatric illnesses. Many behavioral phenotypes have been reported in transgenic and knockout mice, such as learning and memory impairments, feeding disorders, aberrant social, reproductive, parental behaviors, behaviors relevant to human anxiety, depression, schizophrenia, autism, drug addiction, and many others [19-21]. These studies have made important contributions to our knowledge of genetic mechanisms underlying abnormal behaviors in the neuropsychiatric disorders.

In this paper, we report the results of a battery of tests aimed at characterizing behavioral deficits in Dlxl mutant mice. We find that the mutant mice appear normal in their food intake, and in their performance on zero maze and on rotarod tasks; however, they exhibit hyperactivity in open field, in locomotor activity, and in tail suspension tests. Furthermore, they show significantly reduced conditioned fear but no deficits in spontaneous alteration task and spatial working memory. Despite the broad expression of Dlx1 in forebrain GABAergic neurons, Dlx $1^{-/-}$mutants exhibit relatively specific behavioral phenotypes. This analysis suggests that defects in Dlx1 function could contribute to human neuropsychiatric disorders of cognition and emotion.

\section{Materials and methods}

\section{Animals}

Dlxl knockout $\left(D l x 1^{-/-}\right)$, heterozygous $\left(D l x 1^{+/-}\right)$and wildtype $\left(D l x l^{+/+}\right)$mice [22], all age-matched at the time of testing, were maintained on a $\mathrm{C} 57 \mathrm{BL} / 6 \mathrm{~J} \times \mathrm{CD} 1$ background [18]. Wild-type C57BL/6 J mice (The Jackson Laboratory, Bar Harbor, Maine, USA) were used as an additional comparison group for spontaneous alternation and spatial working memory tasks. Mouse colonies were maintained at the Massachusetts Institute of Technology (MIT) or at the University of California, San Francisco (UCSF), in accordance with National Institutes of Health, MIT, and UCSF guidelines for care and use of lab animals.

General behavioral assays were performed on one set of mice (aged 3-6 months), which were all males from three genotype groups $\left(D l x 1^{-/-}, D l x I^{+/-}\right.$and $\left.D l x I^{+/+}\right)$, in the following testing order: zero maze, open field, locomotor activity, food intake, rotarod, and finally tail suspension. We did not perform fear conditioning, prepulse inhibition, or working memory tasks on this set of mice. Mice were group-housed (4-6 mice per cage) in standard polycarbonate mouse cages $(29 \times 18.5 \times 13 \mathrm{~cm})$. They had free access to food and water, and were maintained in a 12 hour light/dark cycle. Between subjects, the testing apparatus was cleaned with a $0.25 \%$ bleach solution, wiped down with water, and dried. With the exception of tests requiring the monitoring of photobeam activity, all animals were tested during the light cycle. Experimental conditions were counterbalanced by genotype.

Multiple sets of the mice (age $\geq 2$ months) were tested for assays in the following order: visual behavioral assays and then fear conditioning (context only, or context and trace). Some sets of these mice were finally tested with prepulse inhibition (aged $\geq 3$ months).

A different set of mice (all males) were tested with the spontaneous alteration task and spatial working memory task. Dlx 1 mice $\left(D l x 1^{+/+}\right.$and $\left.D l x 1^{-/}\right)$tested on these tasks were 11-15 weeks old and group-housed; C57BL/6 J mice 
used as age matched controls were 12-15 weeks old and group-housed. All mice were tested on the spontaneous alteration task first and then on the spatial working memory task 1-2 weeks later. These mice were not tested on any other behavioral assays reported in this manuscript.

For all behavioral tests, investigators were blind to the genotypes. Approximately $3 \mathrm{~min}$ before each assay, the animal was removed from its home cage and placed in a clean holding cage for transfer; an exception to this was the tasks requiring photobeam activity monitoring, where animals were placed directly into the test chambers.

\section{Zero maze}

A zero maze $(34 \mathrm{~cm}$ inner diameter, $46 \mathrm{~cm}$ outer diameter, placed $40 \mathrm{~cm}$ off the ground on 4 braced legs) was used as previously described [23]. Mice were removed and placed in the center of one of the closed quadrants. The test lasted 6 min. Latency in entering (all four paws) an open quadrant, time spent in the open quadrants, and number of closed-to-open quadrant transitions was live-scored in a blinded fashion by a remote observer.

\section{Open field}

A 4-unit open field consisting of a white Kydex box divided into four separate $50 \times 50 \times 38 \mathrm{~cm}$ chambers was used, allowing four animals to be tested concurrently. A video camera was mounted directly above the chambers to monitor the animals' activity and movements. Four mice were simultaneously placed into the open field chambers. A video tracking system (Poly-track, San Diego Instruments, San Diego, CA, USA) was used to measure distance traveled in $5 \mathrm{~min}$ monitoring sessions. Assignments to the four chambers were counterbalanced by genotype. Mouse activity within $7 \mathrm{~cm}$ of the chamber walls was defined as occurring within a peripheral zone; activity further away from the walls was considered to occur within a center zone.

\section{Locomotor activity}

Animals $\left(D l x 1^{+/+}\right.$and $D l x 1^{-/-}$mice; $D l x 1^{+/-}$mice were not tested due to limited availability of activity cages) were housed individually in larger cages $(48 \times 27 \times 13 \mathrm{~cm})$ with bedding, food and water, under a 12-hour light/dark cycle (lights on $7 \mathrm{AM}$ ). To assess activity, we used a photobeam system (FlexField, San Diego Instruments, San Diego, CA, USA), which records horizontal locomotor activity (as monitored by a $4 \times 8$ array of infra-red photobeams). Data were collected for three days, $24 \mathrm{~h}$ each day, with animals placed in the system at noon on day 1 .
Food intake

During the locomotor activity run described above, daily food (standard UCSF laboratory chow pellets) intake measures were made by weighing the food daily.

\section{Rotarod}

Motor coordination was assessed with an Accurotor rotarod machine (Accuscan Instruments, Columbus, Ohio, USA). The rotation rate was accelerated from zero to $50 \mathrm{rpm}$ over 5 min. Four animals were tested concurrently in separate $11 \mathrm{~cm}$-wide compartments, on a rod that was approximately $3 \mathrm{~cm}$ in diameter and was elevated $35 \mathrm{~cm}$. Each animal was assessed over nine trials with $20 \mathrm{~min}$ inter-trial intervals (ITIs). For each trial, the latency to fall from the rod was recorded.

\section{Tail suspension}

Using adhesive tape, animals were suspended by the tail from a $1.2 \mathrm{~cm}$ diameter metal bar elevated $30-35 \mathrm{~cm}$. When suspended, rodents either make "escape attempt" movements, or adopt a characteristic immobile posture. The total time for which the mouse was immobile was measured during a 6 min test period.

\section{Fear conditioning}

All mice were allowed to rest undisturbed in their transportable home cage at least seven days prior to the fear conditioning. To minimize occurrence of handlinginduced seizures in the mutant mice [18], we habituated all animals for 3-5 sessions ( $10 \mathrm{~min}$ in each session, once a day) prior to the day of the first experiment. In these habituation sessions, mice were acclimated to the room and handling of the experimenter. The experimenter wore gloves, habituated the mice to handling, allowing them to walk or run from hand to hand, until the mice showed no apparent signs of stress or fear.

An automated fear-conditioning system (TSE Systems, Bad Homburg, Germany) was used for fear conditioning. Some mice were tested with contextual fear conditioning only (without exposure to a light or tone); the others were tested with both contextual and trace fear conditioning. For each mouse from the $D l x 1^{-/-}, D l x 1^{+/-}$or $D l x 1^{+/+}$groups, training (Day 1) consisted of a $3 \mathrm{~min}$ exposure to the conditioning box (context). In the case of contextual fear conditioning only, this was followed by a foot shock $(2 \mathrm{sec}$, $1.0 \mathrm{~mA}$, constant current). In the trace conditioning sessions, the 3-minute exposure to the context was followed by a visual or auditory cue. In the case of visually cued fear conditioning, the cue was a $30 \mathrm{sec}$ flickering light at $1 \mathrm{~Hz}$, 
followed by a $15 \mathrm{sec}$ trace interval, and then a foot shock (2 sec, $1.0 \mathrm{~mA}$, constant current). Because a visual cue tends to be less effective in eliciting a defensive response than an auditory cue and often requires more conditioned stimulus (CS) - unconditioned stimulus (US) pairings to the light alone [24], the light-pulse-shock sequence was repeated twice before removing the mouse from the box (15 sec ITI). In the case of an auditory cue, after a $3 \mathrm{~min}$ exposure to the box, the mouse was subjected to the tone $(30 \mathrm{sec}, 10 \mathrm{kHz}, 75 \mathrm{~dB}$ sound pressure level (SPL), $200 \mathrm{~ms}$ interval), a $15 \mathrm{sec}$ trace interval, a foot shock (2 sec, $1.0 \mathrm{~mA}$, constant current), and finally a $15 \mathrm{sec}$ interval before removing it from the context. Given a positive correlation of shock intensity (ranging from $0.25 \mathrm{~mA}$ to $1.0 \mathrm{~mA}$ ) with percent of freezing in mice [25], we used a shock intensity of $1.0 \mathrm{~mA}$, which is at the higher end of this range, to ensure that both the wild-type and mutant mice would be conditioned with the foot shock.

The contextual memory test (Day 2) was performed $24 \mathrm{~h}$ after training by re-exposing the mice for $3 \mathrm{~min}$ to the conditioning context. Freezing, defined as a lack of movement except for heart rate and respiration, associated with a crouching posture, was assessed every $10 \mathrm{sec}$, for $180 \mathrm{sec}$, by a trained observer who was unaware of the genotypes. The number of observations indicating freezing was expressed as a percentage of the total number of observations. Baseline freezing for the contextual test refers to freezing (if any) on day 1 during the 3 min exposure to the context, before the tone and foot shock in the training session. We performed the contextual memory test alone, or combined it with the trace conditioning. The results did not differ in the two cases. We report the results on contextual fear conditioning that was carried out alone, i.e. without a visual or auditory cue paired to the shock.

The trace test was performed on day $3,24 \mathrm{~h}$ after the contextual memory test. Mice were placed in a novel context. After a $30 \mathrm{~s}$ habituation period, a $180 \mathrm{~s}$ flickering light $(1 \mathrm{~Hz})$ or a $180 \mathrm{~s}$ tone $(10 \mathrm{kHz}, 75 \mathrm{~dB}$ SPL, $200 \mathrm{~ms}$ interval) was presented in the absence of a foot shock; freezing behavior was recorded every $10 \mathrm{sec}$, over 18 intervals (see above). Baseline freezing for the trace test refers to freezing (if any) on day 3 in the second context, before presenting the light or tone.

Prepulse inhibition

Mice were tested using an ASR-PRO1 acoustic startle reflex test apparatus (Med Associates, St. Albans, VT, USA). Mice were acclimated to the testing room for $1 \mathrm{~h}$ prior to testing. For $2 \mathrm{~min}$ before start of trials, mice were acclimated to the apparatus. Trials were given at an interval of 3-8 s (randomized). Trials consisted of either a $40 \mathrm{~ms}$ startle stimulus alone $(110 \mathrm{~dB}$ white noise $)$ or a startle stimulus preceded $100 \mathrm{~ms}$ earlier by a $20 \mathrm{~ms}$ white noise prepulse at $8 \mathrm{~dB}, 12 \mathrm{~dB}$, or $16 \mathrm{~dB}$ above background (60 $\mathrm{dB}$ white noise). Five trials for each stimulus configuration were recorded using Startle Reflex Software (Med Associates, St. Albans, VT, USA).

\section{Visual cliff test}

Mice were placed in a $60 \times 60 \mathrm{~cm}$ box with a clear Perspex base. A high-contrast grating was attached to the underside of one half of the box; the clear half of the box was placed such that it protruded from the table, revealing a drop to the floor of approximately $90 \mathrm{~cm}$. Mice were placed in the center of the box and their behavior was monitored for $5 \mathrm{~min}$, via a digital video camera mounted above the box. The amount of time spent in each half of the box was recorded. To confirm the role of the visual system in this task, the test was also performed under dark (red light) conditions.

\section{Visual water maze test}

Mice were tested in a circular tank (diameter $1.2 \mathrm{~m}$ ) filled with opaque water. In the center of the target quadrant, a platform $(11 \times 11 \mathrm{~cm})$ was submerged below the water surface, but with a visual cue (a cylinder with high-contrast gratings on the surface) above the water to guide the mouse. The swimming paths of the mice were recorded by a video camera, and analyzed off line by the Videomot 2 software (TSE Systems, Bad Homburg, Germany). Each mouse was placed into the maze from four random points of the tank, and allowed to search for the platform. The amount of time that each mouse took to find the platform was recorded and averaged for comparison.

\section{Spontaneous alternation task (Y-maze)}

Three open arms of an automated radial maze were used to form a Y-maze. Proximal visual cues were attached to the arms and to the hub of the maze to orient the mice in space. C57BL/ $6 \mathrm{~J}, D l x I^{+/+}$and $D l x 1^{-/}$mice were placed in the hub and allowed to traverse the three arms for $8 \mathrm{~min}$. Based on the natural levels of activity in the maze, which peaked at about $2 \mathrm{~min}$, the data were analyzed for the first $2 \mathrm{~min}$, as well as for the entire $8 \mathrm{~min}$ of maze exploration. Alternation was considered to be $\mathrm{ABC}, \mathrm{BCA}$ or $\mathrm{CAB}$, etc, in any order or overlapping occurrence; but not $\mathrm{ABA}$ or $\mathrm{BBC}$. The number of entries into each arm, the percent of alternation ((number of alternations / (total number of arm entries-2)) $\times 100$ ), and the percent of repeat entries into the same arm were calculated.

Spatial working memory task (novel arm in a Y-maze)

For this task, the animal (a C57BL/6 J, Dlx $1^{+/+}$or $D l x 1^{-/-}$ mouse) first explored two randomly selected arms of the Y- 
maze for $5 \mathrm{~min}$, followed by a $2 \mathrm{~min}$ ITI in which the mouse was confined to the center hub of the maze, with all maze arms closed. Next, all three Y-maze arms were opened, and the mouse was free to explore the entire maze for $5 \mathrm{~min}$. The data from the retrieval phase were analyzed to determine if mice recognized the novel arm, expressed by enhanced exploration.

Two measures of novelty exploration/working memory were used to analyze the data from the retrieval phase of this assay: the number of entries into the Y-maze arms and the total amount of time spent in each of the arms.

\section{Statistics}

All $p$ values reported in the results were obtained either from ANOVA for overall comparisons of three or more groups of mice (using genotype as the independent variable) except the repeated measures ANOVA described below, or from t-tests for comparisons between two groups of mice. Locomotor activity of $D l x 1$ mice was analyzed using repeated measures ANOVA with genotype as the between factor, and activity counts over time as the within factor.

Analysis of the prepulse inhibition assay was carried out using repeated measures ANOVA with genotype as the between group variable and prepulse intensity as the within group variable. Where a significant effect was seen between groups of mice, a post-hoc comparison was performed using the Tukey HSD test.

Statistical analysis of the spontaneous alternation task was performed using one-way ANOVA with genotype as the independent variable and number of entries, percent alternation, and percent repeated entries as dependent variables. Data from spatial working memory task were analyzed using repeated measures ANOVA, with genotype as the between variable, number of entries, and time spent in arms as within variables (separate ANOVA for each).

\section{Results}

General activity levels

Our general screen for behavioral phenotypes included the following assays performed on $D l x 1^{-/-}, D l x 1^{+/-}$, and $D l x 1^{+/+}$ mice: zero maze, open field, locomotor activity, food intake, rotarod, and tail suspension.

\section{Zero maze}

No significant differences were detected among the three genotypes on the three measures: open quadrant time, open quadrant entries, and latency to enter open quadrant (data not shown; Dlx $\left.1^{-/-}, \mathrm{n}=7 ; D l x 1^{+/-}, \mathrm{n}=9 ; D l x 1^{+/+}, \mathrm{n}=10\right)$.

\section{Open field}

Overlaid traces for the three genotype groups are presented in Fig. 1a-c. The distance traveled along the periphery of the open field was significantly elevated in Dlx 1 mutants (mean \pm SEM: Dlx $1^{+/+} 1,412 \pm 184 \mathrm{~cm}$; Dlx $1^{+/-} 1,874 \pm 110 \mathrm{~cm} ;$ Dlx $1^{-/-} 2,190 \pm 306 \mathrm{~cm} ; \mathrm{F}(2,21)=$ $3.95, p=0.04$ ) (Fig. 1d). No significant differences were detected among the three groups in center zone entries, time spent in center zone or in periphery, center zone latency, or distance traveled in center zone (data not shown). Also, the percent of time spent in locomotion was significantly enhanced in Dlx 1 mutants (mean \pm SEM: Dlx $1^{+/+} 65.0 \pm 7.2 \% ;$ Dlx $1^{+/-} 78.5 \pm 2.1 \% ;$ D $x 1^{-/-} 86.9 \pm$ $5.3 \% ; \mathrm{F}(2,21)=3.94, p=0.04)$ (Fig. 1e). Thus, the $D l x$ mutants showed locomotor hyperactivity; however, the spatial distribution of this activity did not suggest an anxiety-related phenotype.

\section{Locomotor activity}

We monitored locomotor activity levels for three consecutive days. $D l x 1^{-/}$mice displayed significantly elevated locomotor responses to novel spatial environments compared to their wild-type littermates (main effect of genotype: $\mathrm{F}(1,13)=5.76, p=0.03$; Fig. 2a). Furthermore, total distance traveled was elevated in the $D l x 1^{-/}$group during these $3 \mathrm{~h}$ ( $p=0.02$; Fig. 2b). However, the decreasing activity in the locomotor chamber over time also indicated that both wild-type and Dlx 1 mutants habituate normally, as shown by the main effect of time $(F(1,13)=35.38, p<$ 0.0001; Fig. 2a). Moreover, no difference was detected in their rates of habituation to the novel environment, as shown by the lack of interaction between the genotype and the activity over time $(\mathrm{F}(2,26)=0.96 ; p=0.40$, Fig. $2 \mathrm{a})$. This also indicates that $D l x 1^{-/}$mice, although habituating normally, consistently showed more movement than their wild-type littermates. No differences were seen in the home cage for activity levels or for their circadian regulation.

\section{Food intake}

No differences were detected in daily food intake (or body weight) among the three groups of mice (data not shown; $\left.D l x 1^{-/-}, \mathrm{n}=7 ; D l x 1^{+/-}, \mathrm{n}=9 ; D l x 1^{+/+}, \mathrm{n}=10\right)$.

\section{Rotarod}

No differences among the three groups were recorded in time on the rotarod, indicating no abnormal motor coordination phenotype in the $D l x 1$ mutants (data not shown; Dl $\left.x 1^{-/-}, \mathrm{n}=7 ; D l x 1^{+/-}, \mathrm{n}=9 ; D l x 1^{+/+}, \mathrm{n}=10\right)$. 
Fig. 1 Dlxl mutants displayed locomotor hyperactivity responses in the open field. Representative overlaid traces for the wild-type control (a), $D l x 1^{+/-}$(b), and $D l x 1^{-/-}$(c) groups are presented. Dlxl mutant mice showed significant difference in the mean $( \pm$ SEM $)$ of distance traveled in periphery of open field (d) and percent of time spent in locomotion (e) in the open field test. $D l x I^{+/+}, \mathrm{n}=9$; $D l x 1^{+/-}, \mathrm{n}=9 ; D l x 1^{-/-}, \mathrm{n}=6$. $*=p<0.05$

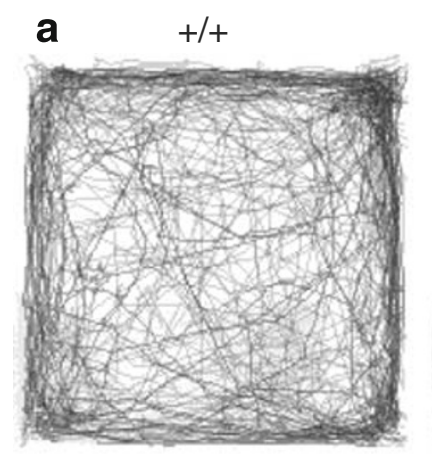

b

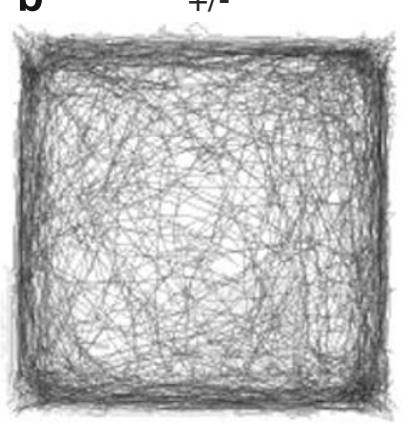

C $\quad-/-$

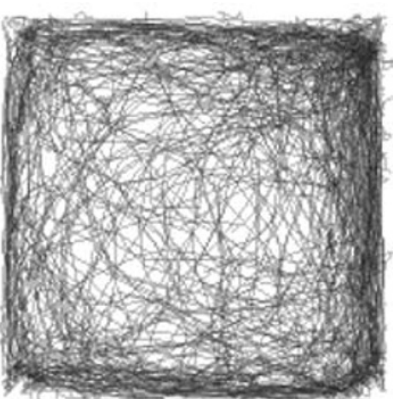

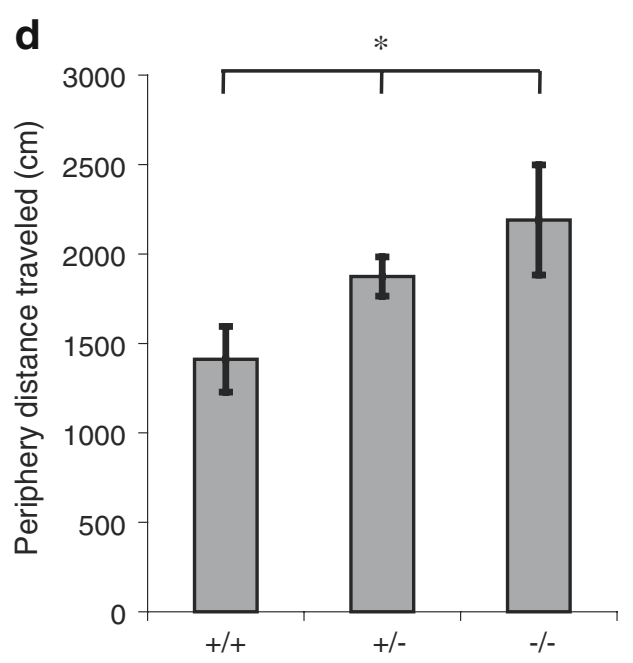

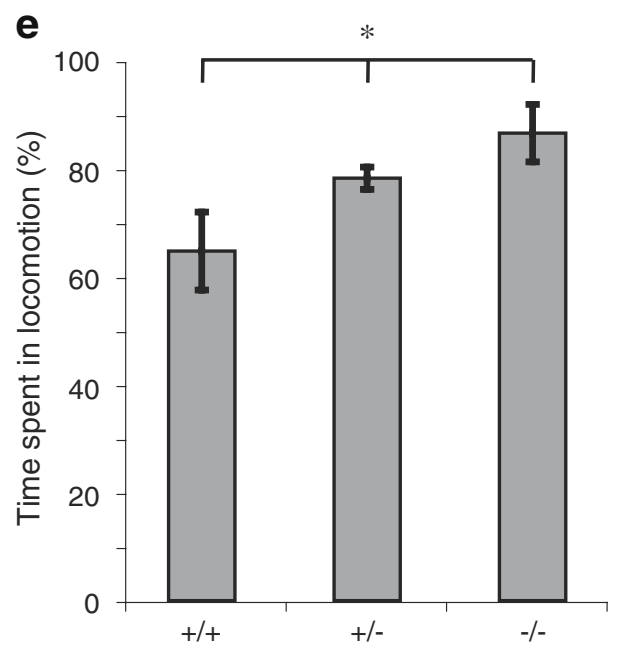

\section{Tail suspension}

As shown in Fig. 3, the mutant mice exhibited reduced immobility times (mean \pm SEM: $D l x 1^{+/+} 76.4 \pm 17.0 \mathrm{~s}, D l x 1^{+/-}$
$34.9 \pm 13.7 \mathrm{~s}$, Dlx $1^{-/-} 28.6 \pm 10.3 \mathrm{~s}$; overall $\mathrm{F}(2,20)=3.19, p=$ $0.06 ; p=0.02$ comparing $D l x 1^{+/+}$and $D l x 1^{-/-}, p=0.04$ comparing $D l x I^{+/+}$and $D l x I^{+/-}$). Dlx $1^{-/-}$mice also showed increased latency to immobility compared to the wild-type

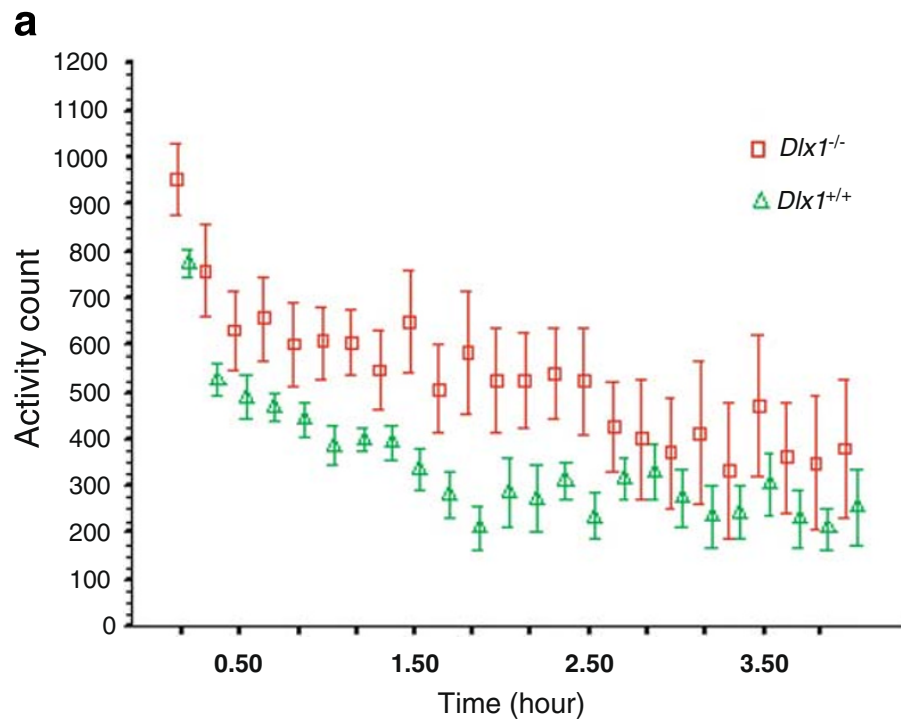

b

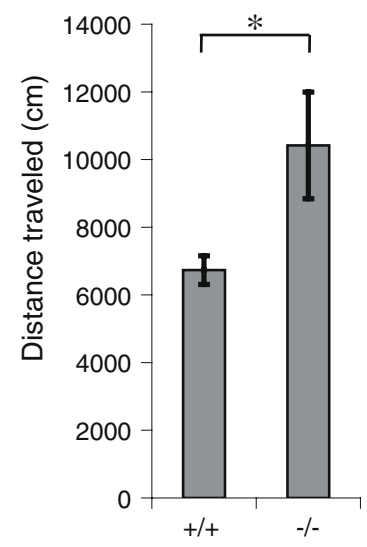

Fig. 2 Dlxl mutant mice exhibited elevated locomotor responses to spatial novelty. (a) Both wild-type and $D l x l^{--}$mice showed normal habituation to the novel environment, but the mutants' activity was consistently elevated, as shown by the mean $( \pm$ SEM) of activity count

during the first $3 \mathrm{~h}$ in the locomotor activity level assay. (b) Locomotion of Dlx 1 mutant mice was significantly elevated, especially in the first $3 \mathrm{~h}$ of activity, as shown by the mean $( \pm$ SEM) of distance that the mice traveled. $D l x I^{+/+}, \mathrm{n}=8 ; D l x I^{-/-}, \mathrm{n}=7 . *=p<0.05$ 
a
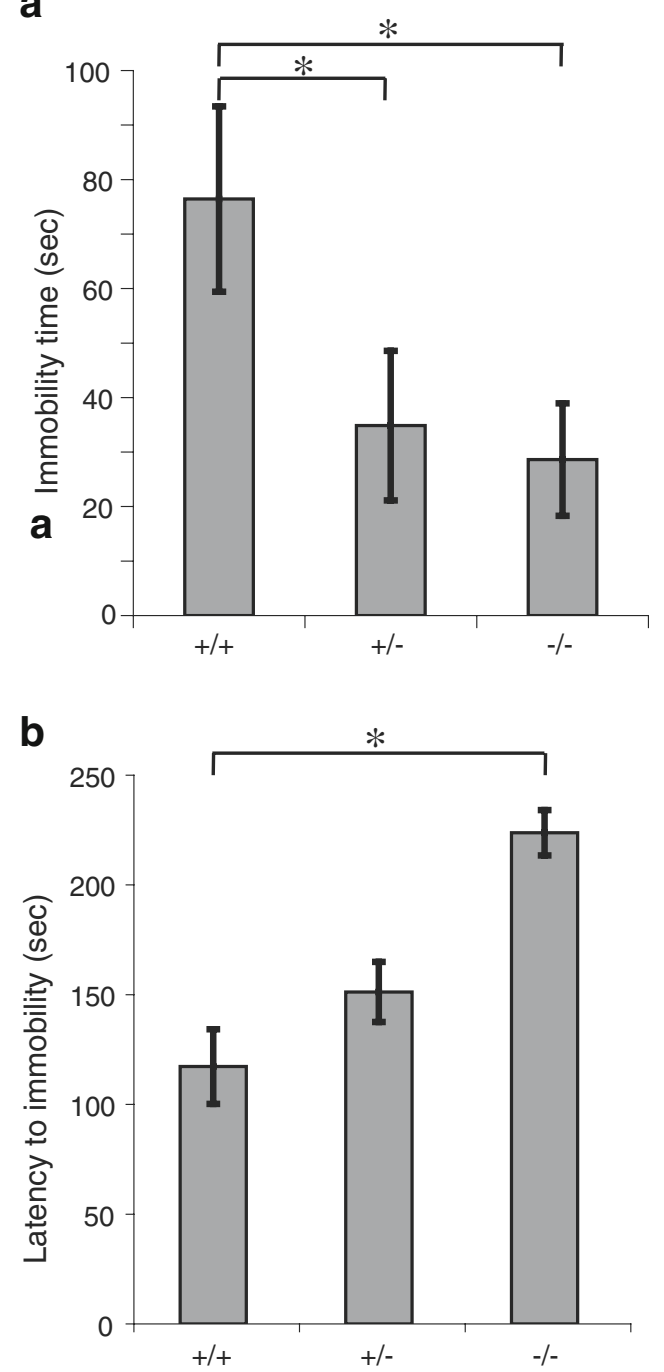

Fig. 3 Dlxl mutants displayed hyperactivity, as indicated by the mean $( \pm$ SEM) of immobility time (a) and latency to immobility (b) in the tail suspension test. $D l x 1^{+/+}, \mathrm{n}=10 ; D l x 1^{+/-}, \mathrm{n}=6 ; D l x 1^{-/-}, \mathrm{n}=7$. $*=p<0.05$

controls (mean \pm SEM: $D l x 1^{+/+} 117.3 \pm 26.5 \mathrm{sec}, D l x 1^{+/-}$ $151.3 \pm 21.3 \mathrm{~s}, D l x 1^{-/-} 223.8 \pm 42.7 \mathrm{~s}$; overall $\mathrm{F}(2,20)=3.07$, $p=0.07 ; p=0.03$ comparing $D l x 1^{+/+}$and $D l x 1^{-/-}, p=0.17$ comparing $D l x I^{+/+}$and $D l x I^{+/-}$).

Fear conditioning

After habituation sessions, each mouse in the $D l x 1^{-/-}$, $D l x I^{+/-}$, and $D l x I^{+/+}$groups received a contextual fear conditioning stimulus alone followed $24 \mathrm{~h}$ later by a contextual fear test, or combined with a trace fear conditioning session (using a light or tone cue) followed $24 \mathrm{~h}$ later by a contextual fear test, followed by a trace fear test after another $24 \mathrm{~h}$. This procedure was performed in four batches of mice consisting of the three genotypes. We did not observe any handling-induced seizures in our fear conditioning assays, including during and after the foot shock.

\section{Baseline freezing}

All mice showed similar amounts of baseline freezing in the apparatus: they displayed virtually no freezing before being subjected to the conditioning in Day 1. The baseline freezing for all three groups was also zero, or close to zero, in Day 3 when the mouse was placed in a new context before being subjected to the visual or auditory cue.

\section{Context}

Mice were shocked once in the context, and tested for fear response $24 \mathrm{~h}$ later. As shown in Fig. $4 \mathrm{a}$, the $D l x l^{-/-}$group exhibited reduced freezing compared to the $D l x I^{+/+}$group (mean \pm SEM: $D l x 1^{+/+} 69.44 \pm 6.84 \%$; $D l x 1^{+/-} 55.89 \pm 8.01 \%$; Dlx $1^{-/-} 20 \pm 4.32 \%$; overall $\mathrm{F}(2,23)=14.08, p=1.02 \times 10^{-4}$; $p=8.90 \times 10^{-5}$ comparing $D l x 1^{+/+}$and $D l x l^{-/-}, p=0.10$ comparing $D l x 1^{+/+}$and $D l x 1^{+/-}$).

We also performed contextual fear conditioning in combination with trace conditioning, and obtained similarly reduced freezing responses in the $D l x l$ mutant mice. For all three groups, when mice were shocked three times during the training, freezing responses to the context were higher than if they were shocked only once (Supplemental Fig. 1), with the $D l x 1^{-/-}$and $D l x 1^{+-}$groups showing trends towards reduced freezing compared to the $D l x 1^{+/+}$ group.

\section{Trace}

Trace fear conditioning [26] is a hippocampus-dependent form of associative learning in which the CS and the US are separated by a time interval; when the CS coterminates or overlaps with the US (as in delay fear conditioning), the associative learning does not involve the hippocampus [2729]. In both cases, the amygdala is required [30].

Because homozygous Dlxl knockout mice $\left(D l x 1^{-/-}\right)$ have been reported to have hearing deficits compared to both heterozygous $\left(D l x I^{+/-}\right)$and wild-type $\left(D l x I^{+/+}\right)$mice [31], we first assessed fear conditioning with a visual cue. $D l x 1^{-/-}$mice showed no overt deficits in vision on visual cliff tests and water maze tests (Supplemental Figs. 2 and 3). Following trace fear conditioning, the $D l x 1^{-/-}$group showed reduced freezing to the light cue relative to the $D l x 1^{+/+}$group (Fig. 4b; mean \pm SEM: $D l x 1^{+/+} 20 \pm 4.18 \%$; Dlx $1^{+/-} 6.48 \pm 2.08 \% ; D l x 1^{-/-} 2.38 \pm 1.19 \%$; overall $\mathrm{F}(2,15)=3.30, p=0.06 ; p=0.02$ comparing $D l x 1^{+/+}$and $D l x 1^{-/-}, p=0.08$ comparing $D l x 1^{+/+}$and $D l x 1^{+/-}$). 
Fig. 4 Lack of $D l x l$ impairs fear conditioning responses in the adult mutant mice. (a) Mean ( \pm SEM) of percent of freezing as a response to the context after fear conditioning (without the light or tone stimulus). In this experiment, one shock after 3-min exposure to the context was administered to elicit the fear response. Baseline freezing before training was zero for all three groups. $D l x 1^{+/+}, \mathrm{n}=6 ; D l x 1^{+/-}, \mathrm{n}=10$; $D l x 1^{-/}, \mathrm{n}=10$. (b) Mean ( $\left.\pm \mathrm{SEM}\right)$ of percent of freezing as a response to the light cue after trace fear conditioning. In this experiment, the light-pulse-shock procedure was performed three times during the training. Baseline freezing before the light cue (in the novel context) was (or close to) zero for all three groups. $D l x 1^{+/+}, \mathrm{n}=6 ; D l x 1^{+/-}, \mathrm{n}=6$; $D l x 1^{-/-}, \mathrm{n}=6$. (c) Mean $( \pm \mathrm{SEM})$ of percent of freezing as a response to the tone after trace fear conditioning. One tone-pulse-shock procedure was performed to elicit the fear response. Baseline freezing before the tone (in the novel context) was (or close to) zero for all three groups. $D l x 1^{+/+}, \mathrm{n}=6 ; D l x 1^{+/,} \mathrm{n}=5 ; D l x 1^{-/-}, \mathrm{n}=10$. For all panels, $*=p<0.05 ; * *=p<0.01$

We obtained similar results in trace fear conditioning using a tone cue (Fig. 4c). Freezing response to the tone was significantly less in $D l x I^{-/-}$and $D l x 1^{+/-}$mice than in $D l x 1^{+/+}$mice (mean \pm SEM: $D l x 1^{+/+} 51.85 \pm 4.74 \%$; $D l x 1^{+/-}$ $22.22 \pm 4.65 \%$; Dlx $1^{-/-} 6.11 \pm 2.21 \%$; overall $\mathrm{F}(2,18)=$ $11.43, p=6.25 \times 10^{-4} ; p=1.03 \times 10^{-3}$ comparing $D l x 1^{+/+}$ and $D l x 1^{-/-}, p=0.03$ comparing $D l x I^{+/+}$and $D l x I^{+/-}$).

Prepulse inhibition

In $D l x 1^{-/-}$mice (no difference in body weight between wildtype and mutant genotypes in this batch of mice), we found possible deficits in prepulse inhibition of acoustic startle response (Fig. 5). Compared to their wild-type littermates, $D l x 1^{-/}$mice showed a trend towards decreased prepulse inhibition at all prepulse intensities that were tested $(8 \mathrm{~dB}$, $12 \mathrm{~dB}$, and $16 \mathrm{~dB}$ above the background of $60 \mathrm{~dB}$ white noise)

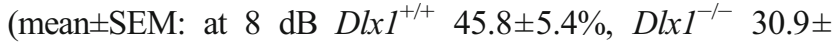
$3.2 \%$; at $12 \mathrm{~dB} D l x I^{+/+} 47.4 \pm 4.9 \%, D l x 1^{-/-} 38.1 \pm 8.7 \%$; at 16 dB $D l x 1^{+/+} 51.2 \pm 3.6 \%, D l x 1^{-/-} 43.1 \pm 7.5 \%$; overall ANOVA $p<0.05)$. A repeated measures ANOVA revealed a significant main effect of genotype $(\mathrm{F}(1,69)=4.07, p<0.05)$ and no

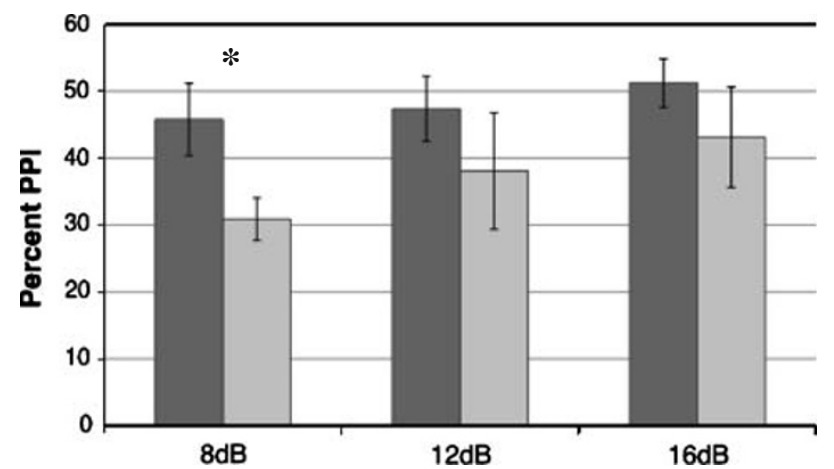

Fig. $5 \mathrm{Dl} x \mathrm{I}^{-/-}$mice showed reduced prepulse inhibition of the acoustic startle response. Analysis was performed at 8, 12, and $16 \mathrm{~dB}$ above background. $*=p<0.05 . D l x 1^{+/+}, \mathrm{n}=16$ (8 females); $D l x 1^{-/-}$, $\mathrm{n}=9$ (5 females). Dark bars: $D l x 1^{+/+}$; light bars: $D l x 1^{-}$
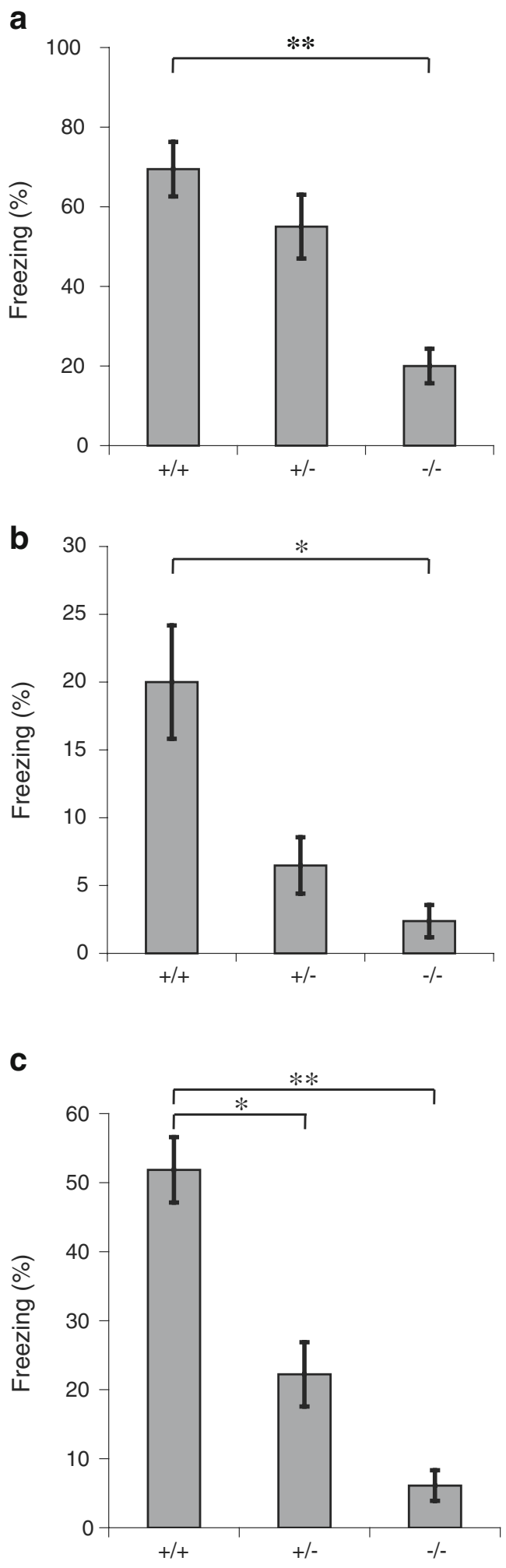

significant main effect of prepulse intensity $(\mathrm{F}(2,69)=0.79$, $p=0.46$ ) or interaction between genotype and prepulse intensity $(\mathrm{F}(2,138)=0.15, p=0.86)$. A post-hoc comparison showed a significant difference between genotypes at $8 \mathrm{~dB}$ $(p<0.05)$, but not at $12 \mathrm{~dB}$ or $16 \mathrm{~dB}$ above background. 
Because this decreased attenuation of the acoustic startle reflex could reflect changes in sensorimotor gating [32] and/or a hearing deficit [31], we analyzed the startle reflex amplitude when no prepulse stimulus was present (Supplemental Fig. 4). The startle response (without a prepulse) at $110 \mathrm{~dB}$ was significantly lower for $D l x 1^{-/-}$mice than $D l x 1^{+/+}$mice (mean \pm SEM in arbitrary units: Dl $x 1^{+/+}$ $\left.920.43 \pm 74.90, D l x 1^{-1-} 630.76 \pm 105.91 ; p=0.04\right)$. This finding is consistent with a modest hearing deficit in the Dlx $1^{-/-}$mice [31].

\section{Spontaneous alternation task (Y-maze)}

In light of the deficits in fear conditioning in the $D l x 1^{-/-}$ mice, we also investigated whether these animals had abnormalities in processes such as working memory. The spontaneous alternation task is based on the natural alternation behavior of rodents, and is used to model spatial working memory with a delay time of zero seconds [33]. $D l x 1^{-/-}$mice performed indistinguishably from their wildtype littermates, and at the same level as C57BL/6 J mice, on this task: the number of entries into maze arms, the percent of alternations ((number of alternations / (total number of arm entries-2) $\times 100$ ), and the percent of repeat entries were similar among these three groups for the first $2 \mathrm{~min}$, as well as for the entire $8 \mathrm{~min}$ (Supplemental Fig. 5). The results from one-way ANOVA on the above data are summarized in supplemental Table 1.

Performance of a complete alternation on a Y-maze at chance levels has not been found reported in literature. However, if determined mathematically (calculated using probabilities of entries), the chance level of alternation would be $22.22 \%$. All mice tested on this task have performed higher than this level of alternation.

Spatial working memory task (novel arm in a Y-maze)

A two-trial procedure, originally developed to test novelty response and working memory in a Y-maze, was applied to test spatial memory and to determine exploration of novel locations [34]. We used a 2 min interval between trials, to correspond with the limited time span considered to be working memory. This task is known to tax the dorsolateral prefrontal cortex (PFC) in primates, and medial PFC in rodents [35-37]. Proximal visual cues were attached to the arms and to the hub of the maze to orient the mice in space. As indicated by the number of entries into the arms, and the total amount of time spent in each of the arms (Supplemental Fig. 6), Dlx $1^{+/+}$and $D l x 1^{-/-}$mice, as well as C57BL/6 J mice, recognized novel locations, and showed significantly enhanced exploration of novel locations after the 2 min ITI. Thus, the $D l x 1^{-/}$mutants behaved normally in this assay.
Using repeated measures ANOVA on the number of entries (Supplemental Fig. 6a), we found that main effect of entries was observed for all groups of mice $(\mathrm{F}(2,29)=48.87$, $p<0.0001$ ), indicating that all mice explored the novel arm significantly more than the other arms. However, no difference was detected among the mouse groups in the overall level of entries into maze arms (no genotype effect: $\mathrm{F}(2,29)=2.44, p=0.11)$. Furthermore, all mice showed similar preference for the novel arm entries over the entries to the other arms, as shown by lack of interaction between genotype and number of entries $(\mathrm{F}(4,58)=1.98, p=0.11)$.

Using repeated measures ANOVA on the time spent in arms (Supplemental Fig. 6b), we found that main effect of time spent in arms was observed for all groups of mice, indicating strong preference for spending time in the novel $\operatorname{arm}(\mathrm{F}(2,29)=22.56, p<0.0001)$. No differences were observed between any of the groups in time spent in maze arms (no genotype effect: $\mathrm{F}(2,29)=1.24, p=0.30$ ). All groups of mice consistently spent more time in the novel arm, as shown by lack of interaction between genotype and time spent in different maze arms $(\mathrm{F}(4,58)=0.61$, $p=0.66)$.

In Supplemental Fig. 6c, the novel arm proportions (percent of time spent in novel arm compared to the other two arms) were analyzed using one-sample t-tests comparing the mean of novel arm proportion to the hypothetical $33.33 \%$ mean, which would be the "random" proportion for the novel arm. For the percent of novel entries compared to entries into the other two arms, all three groups showed significantly enhanced exploration of the novel arm (C57BL/6 J: $p<0.0001 ; D l x 1^{+/+}: p=0.03 ; D l x 1^{-/-}: p=$ $0.01)$. Similar results were found for the percent of time spent in the novel arm compared to in the other two arms (C57BL/6 J: $p<0.0001 ; D l x 1^{+/+}: p=0.03 ;$ Dlx $1^{-/-}$: $p=0.13)$.

\section{Discussion}

We found that lack of Dlxl leads to deficits in fear conditioning and possibly in prepulse inhibition in $D l x l$ mutant mice. The Dlxl mutants display hyperactivity in tests of open field, locomotor activity, and tail suspension, but they appear normal in other aspects of general activities and coordination such as food intake, zero maze and rotarod performances. Nonetheless, this phenotype shows specificity, as the mutant mice exhibit normal performance in tasks related to working memory.

Forebrain neural systems mediating fear conditioning

Pavlovian fear conditioning assays are effective at assessing associative learning. Thus, the anomaly in fear learning of 
the Dlxl mutants could be secondary to learning deficits. Multiple neural circuits, including those interconnecting the amygdala, hippocampus and PFC, could contribute to their reduction in fear conditioning.

The neural hub for fear conditioning is the amygdala [30]. Sensory stimuli corresponding to the potential CSs from the thalamus, hippocampus, and several cortical regions arrive at the the lateral nucleus of the basolateral complex. The primary output for the generation of conditional fear responses is the central nucleus of the amygdala, which projects to a wide range of regions responsible for emotional responses [30].

The hippocampus is another key component of the fear conditioning system. In contextual fear conditioning, the hippocampus is important for multimodal contextual cues, but is not necessary for conditioning to a discrete unimodal stimulus, such as a tone [38-40]. Furthermore, the hippocampus has been implicated in contextual memory retrieval [41]. Muscimol inactivation of the dorsal hippocampus $(\mathrm{DH})$ impairs contextual retrieval of fear memory [42]. Also, inactivation of the ventral hippocampus (VH) impairs memory for context [43]. In trace fear conditioning, the $\mathrm{DH}$ is required for normal function [44, 45]. Exposure to a trace-conditioned cue increases immediate early gene expression in the dentate gyrus [46]. Trace conditioning also increases neurogenesis in the dentate gyrus [47], and is impaired by a reduction in newly generated neurons in this region [48].

Finally, the PFC has a critical role in fear conditioning. Trace fear conditioning increases extracellular signalregulated kinase (ERK) activation in the medial prefrontal cortex (mPFC) immediately following training, and blocking this increase impairs memory tested $48 \mathrm{~h}$ later [49]. Studies on trace fear conditioning with intra-mPFC infusion of muscimol indicated that the $\mathrm{mPFC}$ activity is required for both recent and remote memory recall [50]. Trace conditioning is associated with increased neuronal activity (c-fos expression) in the anterior cingulate cortex, and is selectively impaired by lesions of this structure. While we did not find evidence indicating working memory-related defects in the PFC of Dlxl mutants, it is formally possible that abnormal functioning of the mPFC contributed to their fear conditioning phenotype.

Furthermore, PFC function, which is important for spatial working memory tasks, requires continuously keeping spatial information in memory for short (seconds to few minutes) durations. Interneurons in the PFC have a critical role in this process [51-53]. Specifically, it has been shown that parvalbumin + interneurons are important for working memory [54, 55]. The putatively normal parvalbumin-containing interneurons [18] may contribute to the unaltered performance of $D l x 1^{-/}$mutants in the working memory tasks described in this report.
Forebrain neural systems mediating behavioral inhibition

Dlx 1 mutants also display generalized elevations of activity levels, consistent with deficits in behavioral inhibition. It is possible that such deficits in behavioral inhibition could contribute to reduction of freezing in the fear conditioning assays. Additional studies will be required to determine the relative contributions of behavioral inhibition and associative learning defects to this phenotype. Connections from the PFC to the basal ganglia and cerebellum have been implicated in modulating behavioral inhibition [56, 57]. An impaired ability to inhibit behavior leads to hyperactivity [58], impulsivity [59], perseveration [60], and obsessive/compulsive behavior [59], and is associated with many neuropsychiatric disorders. To understand the anatomical substrates where lack of $D l x l$ function contributes to these behaviors, it would be necessary to selectively remove $D l x 1$ function in the different components of these circuits.

Forebrain neural systems mediating prepulse inhibition

Prepulse inhibition is commonly used as a measure of the sensorimotor gating of the startle reflex [32]. Similar to fear conditioning, prepulse inhibition is regulated by multiple forebrain components, in addition to subcortical regions that process hearing and mediate motor responses (including the cochlea, inferior colliculus and pons). Within the forebrain, the cortico-basal ganglia-thalamic circuit is thought to have a central function, with the output through a pathway from the medial prefrontral cortex, accumbens and ventral pallidum to the pons. Inputs to the accumbens from the amygdala, hippocampus and ventral tegmental area also impact this system [32].

Dlx1 is expressed in the above circuits that mediate prepulse inhibition (see below), providing possible explanation for the reduced prepulse inhibition that we observed in the Dlxl mutants. However, this finding needs to be interpreted with caution. The $D l x$ genes are expressed in the developing middle ear; $D l x 1^{-/}$mutants have dysmorphic ossicles and reduced hearing [31]. These deficits in auditory ability of the mutant mice could result in the small changes in their acoustic startle amplitudes and prepulse inhibition.

Despite the reduced single-stimulus startle reflex amplitude in these mice, the observation that Dlx 1 mutant mice do show inhibition of startle responses indicates that they are capable of hearing the prepulse stimulus, and suggests that there may indeed be deficits in prepulse inhibition in these mutant mice. For further investigation of this phenotype, prepulse inhibition using cross-modal stimuli (e.g. light) would be useful. 
Dlxl expression and function in forebrain neural systems

The Dlx genes are probably expressed at some point during development in all forebrain GABAergic neurons, and not in GABAergic neurons in other parts of the central nervous system [16, 61-63]. This includes local circuit neurons of the neocortex, hippocampus and amygdala, projection neurons of the basal ganglia (striatum, accumbens, pallidum, septum and central nucleus of the amygdala), and inhibitory neurons of the thalamus (reticular nucleus). Dlx 1 is broadly expressed prenatally in these regions. In cortical regions, including the PFC, hippocampus and basolateral and lateral amygdala, $D l x 1$ is expressed primarily in dendrite-innervating interneurons $[18,64]$. In addition, $D l x 1$ is expressed in the medial amygdala, and perhaps the intercalated nuclei (Wang and Rubenstein, unpublished observations). Thus, Dlxl could impact many of the key components of the forebrain neural systems that regulate fear conditioning, prepulse inhibition, and behavioral inhibition.

Furthermore, Dlxl has critical roles in regulating the physiology of the neocortex and hippocampus through promoting inhibitory tone and maintaining interneuron survival [18]. Approximately 30 days after birth, Dlx $1^{-/-}$ mice begin to specifically lose somatostatin+, calretinin+, and NPY+ interneuron subtypes [18], which primarily target dendrites $[1,65]$. Around this time, the mutant mice have increased susceptibility to seizures [18].

Currently, there is little information about whether $D l x 1^{-/-}$mutants have anatomical or physiological defects in other regions of the forebrain where $D l x 1$ is expressed. While they show reduced numbers of dopaminergic neurons in the arcuate nucleus of the hypothalamus (Yee, Wang, Ekker, Anderson and Rubenstein, submitted), future analyses of additional $D l x 1^{-/}$forebrain components could be important in elucidating their abnormal behaviors.

\section{Role of GABAergic signaling in fear conditioning}

Similar to the $D l x 1^{-/-}$mutants, other mouse models with altered inhibitory systems show changes in fear conditioning and general activity. However, unlike the $D l x 1^{-/}$mice, many of these show increased indices of fearfulness. For example, $G A B A_{A}$ receptor $\beta 3$ subunit $^{-/}$mice have epilepsy, hyperactivity, hypersensitivity to human contact and other sensory stimuli, and lack of coordination [66]. $G A B A_{A}$ receptor $\gamma 2^{+/-}$mice showed enhanced behavioral inhibition toward natural aversive stimuli, heightened responsiveness in trace conditioning and ambiguous cue discrimination learning, and unaffected contextual and delay conditioning [67]. Mice with a point mutation in the $\alpha 5 G A B A_{A}$ receptor gene, which renders the receptor diazepam-insensitive, have selective reduction of $\alpha 5 \mathrm{GABA}_{\mathrm{A}}$ receptors only in the hippocampal pyramidal cells and show facilitated trace conditioning but not delay or contextual conditioning [68]. GABA ${\text { receptor } \delta \text { subunit }^{-/}}^{-}$ mice have reduced synaptic and extrasynaptic inhibition and decreased sensitivity to neurosteroids [69-72], and exhibit enhanced acquisition of tone and context fear [73].

While these GABA receptor mutants generally show increased fear, the $D l x 1^{-/-}$mutants have reduced fear conditioning. Perhaps this difference is because the receptor mutants affect most types of inhibitory synapses, whereas the Dlx 1 mutation appears to selectively affect dendriteinnervating synapses. Thus, future studies are needed to establish the mechanism(s) underlying the reduced fear conditioning of $D l x 1^{-/}$mutants, with a particular focus on dissecting the role of $D l x$ in the PFC, hippocampus and amygdala. Finally, our results raise the possibility that selective defects in subsets of forebrain GABAergic neurons could contribute to human disorders of emotional dysregulation and hyperactivity. This opens the door to considering the possibility that Dlxl dosage in humans could modulate fear conditioning, as well as the assessment and response to danger.

Altered inhibitory systems have been implicated in multiple brain disorders. Given the critical roles of Dlx 1 in interneuron development and function and the deficits in interneurons in Dlxl mutant mice [18], the behavioral abnormalities in these mutants provide evidence for a link between specific components of the GABAergic system and specific behavioral phenotypes. Thorough investigations of mechanisms underlying these behavioral phenotypes in Dlxl mutants may provide further understanding on contributions of GABAergic inhibition in complex human neuropsychiatric diseases.

Acknowledgements This work was supported by funds to M.S. (Simons Foundation and the Autism Consortium), R.M. (National Eye Institute Kirschstein-NRSA Fellowship 1 F32 EY017243), D.T.P. (Nancy Lurie Marks Family Foundation), P.H.J. (funds from the state of California), and J.L.R.R. (Nina Ireland and NIMH R37 Grant MH049428). We thank Jed Holtzman and Luna Abdallah for assistance in studies on mouse general activity levels, Li-Huei Tsai, Ji-Song Guan, and Andre Fischer for advice and assistance in fear conditioning assays, Orsolya Kuti for help in maintaining mouse colonies, Yuri Ostrovsky and Cortina McCurry for insightful discussion on data analysis. We are grateful to Sonal Jhaveri for valuable comments on the manuscript.

Open Access This article is distributed under the terms of the Creative Commons Attribution Noncommercial License which permits any noncommercial use, distribution, and reproduction in any medium, provided the original author(s) and source are credited.

\section{References}

1. Markram $\mathrm{H}$, et al. Interneurons of the neocortical inhibitory system. Nat Rev Neurosci. 2004;5(10):793-807. 
2. Ascoli GA, et al. Petilla terminology: nomenclature of features of GABAergic interneurons of the cerebral cortex. Nat Rev Neurosci. 2008;9(7):557-68.

3. Lewis DA, Hashimoto T, Volk DW. Cortical inhibitory neurons and schizophrenia. Nat Rev Neurosci. 2005;6(4):312-24.

4. Lewis DA, Gonzalez-Burgos G. Neuroplasticity of neocortical circuits in schizophrenia. Neuropsychopharmacology. 2008;33 (1):141-65.

5. Acosta MT, Pearl PL. The neurobiology of autism: new pieces of the puzzle. Curr Neurol Neurosci Rep. 2003;3(2):149-56.

6. Rubenstein JL, Merzenich MM. Model of autism: increased ratio of excitation/inhibition in key neural systems. Genes Brain Behav. 2003;2(5):255-67.

7. Mohler H, Fritschy JM, Rudolph U. A new benzodiazepine pharmacology. J Pharmacol Exp Ther. 2002;300(1):2-8.

8. Freund TF. Interneuron Diversity series: Rhythm and mood in perisomatic inhibition. Trends Neurosci. 2003;26(9):489-95.

9. Brambilla $\mathrm{P}$, et al. GABAergic dysfunction in mood disorders. Mol Psychiatry 2003; 8(8): p. 721-37, 715 .

10. Tunnicliff G, Malatynska E. Central GABAergic systems and depressive illness. Neurochem Res. 2003;28(6):965-76.

11. Choudary PV, et al. Altered cortical glutamatergic and GABAergic signal transmission with glial involvement in depression. Proc Natl Acad Sci U S A. 2005;102(43):15653-8.

12. Anderson $\mathrm{SA}$, et al. Interneuron migration from basal forebrain to neocortex: dependence on Dlx genes. Science. 1997;278 (5337):474-6.

13. Anderson SA, et al. Mutations of the homeobox genes Dlx-1 and Dlx-2 disrupt the striatal subventricular zone and differentiation of late born striatal neurons. Neuron. 1997;19(1):27-37.

14. Marin O, Anderson SA, Rubenstein JL. Origin and molecular specification of striatal interneurons. J Neurosci. 2000;20 (16):6063-76.

15. Panganiban G, Rubenstein JL. Developmental functions of the Distal-less/Dlx homeobox genes. Development. 2002;129 (19):4371-86.

16. Stuhmer T, et al. Expression from a Dlx gene enhancer marks adult mouse cortical GABAergic neurons. Cereb Cortex. 2002;12 (1):75-85.

17. Yun K, et al. Modulation of the notch signaling by Mash1 and Dlx $1 / 2$ regulates sequential specification and differentiation of progenitor cell types in the subcortical telencephalon. Development. 2002;129(21):5029-40.

18. Cobos I, et al. Mice lacking Dlx1 show subtype-specific loss of interneurons, reduced inhibition and epilepsy. Nat Neurosci. 2005;8(8):1059-68.

19. Crawley JN. Behavioral phenotyping of transgenic and knockout mice: experimental design and evaluation of general health, sensory functions, motor abilities, and specific behavioral tests. Brain Res. 1999;835(1):18-26.

20. Crawley JN. Mouse behavioral assays relevant to the symptoms of autism. Brain Pathol. 2007;17(4):448-59.

21. Ricceri L, Moles A, Crawley J. Behavioral phenotyping of mouse models of neurodevelopmental disorders: relevant social behavior patterns across the life span. Behav Brain Res. 2007;176(1):40-52.

22. Qiu M, et al. Role of the Dlx homeobox genes in proximodistal patterning of the branchial arches: mutations of Dlx-1, Dlx-2, and Dlx-1 and -2 alter morphogenesis of proximal skeletal and soft tissue structures derived from the first and second arches. Dev Biol. 1997;185(2):165-84.

23. Heisler LK, et al. Elevated anxiety and antidepressant-like responses in serotonin 5-HT1A receptor mutant mice. Proc Natl Acad Sci U S A. 1998;95(25):15049-54.

24. Heldt $\mathrm{S}$, et al. Posttraining lesions of the amygdala interfere with fear-potentiated startle to both visual and auditory conditioned stimuli in C57BL/6 J mice. Behav Neurosci. 2000;114(4):749-59.
25. Anagnostaras SG, et al. Computer-assisted behavioral assessment of Pavlovian fear conditioning in mice. Learn Mem. 2000;7 (1):58-72.

26. Pavlov I. Conditioned reflexes: an investigation of the physiological activity of the cerebral cortex. London: Oxford UP; 1927.

27. Ross RT, et al. Hippocampectomy disrupts acquisition and retention of learned conditional responding. Behav Neurosci. 1984;98(2):211-25.

28. Paulsen O, Moser EI. A model of hippocampal memory encoding and retrieval: GABAergic control of synaptic plasticity. Trends Neurosci. 1998;21(7):273-8.

29. Wallenstein GV, Eichenbaum H, Hasselmo ME. The hippocampus as an associator of discontiguous events. Trends Neurosci. 1998;21(8):317-23.

30. Fanselow MS and Poulos AM. The neuroscience of mammalian associative learning. Annu Rev Psychol 2005; 56: p. 207-34.

31. Polley DB, et al. Severe hearing loss in Dlxl mutant mice. Hear Res. 2006;214(1-2):84-8.

32. Swerdlow NR, Braff DL, Geyer MA. Animal models of deficient sensorimotor gating: what we know, what we think we know, and what we hope to know soon. Behav Pharmacol. 2000;11(34):185-204.

33. Lalonde R. The neurobiological basis of spontaneous alternation. Neurosci Biobehav Rev. 2002;26(1):91-104.

34. Dellu F, et al. Genetic differences in response to novelty and spatial memory using a two-trial recognition task in mice. Neurobiol Learn Mem. 2000;73(1):31-48.

35. Kolb B. Functions of the frontal cortex of the rat: a comparative review. Brain Res. 1984;320(1):65-98.

36. Goldman-Rakic PS. Cellular and circuit basis of working memory in prefrontal cortex of nonhuman primates. Prog Brain Res 1990; 85: p. 325-35; discussion 335-6.

37. Ongur D, Price JL. The organization of networks within the orbital and medial prefrontal cortex of rats, monkeys and humans. Cereb Cortex. 2000;10(3):206-19.

38. Kim JJ, Fanselow MS. Modality-specific retrograde amnesia of fear. Science. 1992;256(5057):675-7.

39. Phillips RG, LeDoux JE. Differential contribution of amygdala and hippocampus to cued and contextual fear conditioning. Behav Neurosci. 1992;106(2):274-85.

40. Anagnostaras SG, Maren S, Fanselow MS. Temporally graded retrograde amnesia of contextual fear after hippocampal damage in rats: within-subjects examination. J Neurosci. 1999;19(3):1106-14.

41. Hirsh R. The hippocampus and contextual retrieval of information from memory: a theory. Behav Biol. 1974;12(4):421-44.

42. Holt W, Maren S. Muscimol inactivation of the dorsal hippocampus impairs contextual retrieval of fear memory. J Neurosci. 1999;19(20):9054-62.

43. Rudy JW, Matus-Amat P. The ventral hippocampus supports a memory representation of context and contextual fear conditioning: implications for a unitary function of the hippocampus. Behav Neurosci. 2005;119(1):154-63.

44. McEchron MD, et al. Hippocampectomy disrupts auditory trace fear conditioning and contextual fear conditioning in the rat. Hippocampus. 1998;8(6):638-46.

45. Quinn JJ, et al. Post-training excitotoxic lesions of the dorsal hippocampus attenuate forward trace, backward trace, and delay fear conditioning in a temporally specific manner. Hippocampus. 2002;12(4):495-504.

46. Weitemier AZ, Ryabinin AE. Subregion-specific differences in hippocampal activity between Delay and Trace fear conditioning: an immunohistochemical analysis. Brain Res. 2004;995(1):55-65.

47. Gould E, et al. Learning enhances adult neurogenesis in the hippocampal formation. Nat Neurosci. 1999;2(3):260-5.

48. Shors TJ, et al. Neurogenesis in the adult is involved in the formation of trace memories. Nature. 2001;410(6826):372-6. 
49. Runyan JD, Moore AN, Dash PK. A role for prefrontal cortex in memory storage for trace fear conditioning. J Neurosci. 2004;24 (6): 1288-95.

50. Blum S, Hebert AE, Dash PK. A role for the prefrontal cortex in recall of recent and remote memories. Neuroreport. 2006;17(3):341-4.

51. Sawaguchi T, Goldman-Rakic PS. The role of D1-dopamine receptor in working memory: local injections of dopamine antagonists into the prefrontal cortex of rhesus monkeys performing an oculomotor delayed-response task. J Neurophysiol. 1994;71(2):515-28.

52. Rao SG, Williams GV, Goldman-Rakic PS. Destruction and creation of spatial tuning by disinhibition: $\operatorname{GABA}(\mathrm{A})$ blockade of prefrontal cortical neurons engaged by working memory. J Neurosci. 2000;20(1):485-94.

53. Sawaguchi T, Iba M. Prefrontal cortical representation of visuospatial working memory in monkeys examined by local inactivation with muscimol. J Neurophysiol. 2001;86(4):2041-53.

54. Williams SM, Goldman-Rakic PS, Leranth C. The synaptology of parvalbumin-immunoreactive neurons in the primate prefrontal cortex. J Comp Neurol. 1992;320(3):353-69.

55. Wang XJ, et al. Division of labor among distinct subtypes of inhibitory neurons in a cortical microcircuit of working memory. Proc Natl Acad Sci U S A. 2004;101(5):1368-73.

56. Barkley RA. Attention-deficit/hyperactivity disorder, selfregulation, and time: toward a more comprehensive theory. J Dev Behav Pediatr. 1997;18(4):271-9.

57. Brennan AR and Arnsten AF. Neuronal mechanisms underlying attention deficit hyperactivity disorder: the influence of arousal on prefrontal cortical function. Ann N Y Acad Sci 2008; 1129: p. 236-45.

58. Barkley RA. Behavioral inhibition, sustained attention, and executive functions: constructing a unifying theory of ADHD. Psychol Bull. 1997;121(1):65-94.

59. Chamberlain SR, Sahakian BJ. The neuropsychiatry of impulsivity. Curr Opin Psychiatry. 2007;20(3):255-61.

60. Hauser MD. Perseveration, inhibition and the prefrontal cortex: a new look. Curr Opin Neurobiol. 1999;9(2):214-22.

61. Stuhmer T, et al. Ectopic expression of the Dlx genes induces glutamic acid decarboxylase and Dlx expression. Development. 2002;129(1):245-52.
62. Kohwi M, et al. A subpopulation of olfactory bulb GABAergic interneurons is derived from Emx1- and Dlx5/6-expressing progenitors. J Neurosci. 2007;27(26):6878-91.

63. Potter GB, et al. Generation of Cre-transgenic mice using Dlx1/ Dlx2 enhancers and their characterization in GABAergic interneurons. Mol Cell Neurosci 2008.

64. Cobos I, et al. Cellular patterns of transcription factor expression in developing cortical interneurons. Cereb Cortex. 2006;16(Suppl 1):i82-8.

65. DeFelipe J. Neocortical neuronal diversity: chemical heterogeneity revealed by colocalization studies of classic neurotransmitters, neuropeptides, calcium-binding proteins, and cell surface molecules. Cereb Cortex. 1993;3(4):273-89.

66. Homanics GE, et al. Mice devoid of gamma-aminobutyrate type A receptor beta3 subunit have epilepsy, cleft palate, and hypersensitive behavior. Proc Natl Acad Sci U S A. 1997;94(8):4143-8.

67. Crestani F, et al. Decreased GABAA-receptor clustering results in enhanced anxiety and a bias for threat cues. Nat Neurosci. 1999;2 (9):833-9.

68. Crestani F, et al. Trace fear conditioning involves hippocampal alpha5 GABA(A) receptors. Proc Natl Acad Sci U S A. 2002;99 (13):8980-5.

69. Mihalek RM, et al. Attenuated sensitivity to neuroactive steroids in gamma-aminobutyrate type A receptor delta subunit knockout mice. Proc Natl Acad Sci U S A. 1999;96(22):12905-10.

70. Vicini S, Losi G, Homanics GE. GABA(A) receptor delta subunit deletion prevents neurosteroid modulation of inhibitory synaptic currents in cerebellar neurons. Neuropharmacology. 2002;43 (4):646-50.

71. Porcello DM, et al. Intact synaptic GABAergic inhibition and altered neurosteroid modulation of thalamic relay neurons in mice lacking delta subunit. J Neurophysiol. 2003;89(3):137886.

72. Spigelman I, et al. Reduced inhibition and sensitivity to neurosteroids in hippocampus of mice lacking the $\operatorname{GABA}(\mathrm{A})$ receptor delta subunit. J Neurophysiol. 2003;90(2):903-10.

73. Wiltgen BJ, et al. Trace fear conditioning is enhanced in mice lacking the delta subunit of the GABAA receptor. Learn Mem. 2005;12(3):327-33. 